J Mol Biol. 2009 October 9; 392(5): 1133-1144. doi:10.1016/j.jmb.2009.07.050.

\title{
One is Not Enough
}

\author{
Robert Daber, Kim Sharp, and Mitchell Lewis $\ddagger$ \\ Department of Biochemistry and Biophysics, University of Pennsylvania School of Medicine, 37th \\ and Hamilton Walk, Philadelphia, Pennsylvania 19104-6059
}

\section{Summary}

In both prokaryotic and eukaryotic organisms, repressors and activators are responsible for regulating gene expression. The lac operon is a paradigm for understanding how metabolites function as signaling molecules and modulate transcription. These metabolites or allosteric effector molecules bind to the repressor and alter the conformational equilibrium between the induced and the repressed states. Here we describe a set of experiments where we modified a single inducer binding site in a dimeric repressor and examined its effect on induction. Based upon these observations, we have been able to calculate the thermodynamic parameters that are responsible for the allosteric properties that govern repressor function. Understanding how effector molecules alter the thermodynamic properties of the repressor is essential for establishing a detailed understanding of gene regulation.

\section{Keywords}

lac repressor; allostery; induction; repression; inducer binding

\section{Introduction}

Cellular proteins that perform specific metabolic tasks are often regulated to meet the needs of the organism. In many instances, regulating the flux through a pathway is achieved by adjusting the concentration of an enzyme that controls the rate determining step in a pathway. In both prokaryotic and eukaryotic organisms, enzyme concentration is regulated by transcription, which is frequently controlled by repressors and activators. These molecules either directly or indirectly monitor the accumulation or diminution of a metabolite and respond like a molecular switch, increasing or decreasing the rate of gene expression. Effector molecules are the chemical signals that convey the metabolic state of the cell to the genetic machinery. They can function as inducers, anti-inducers or co-repressors and in each instance they alter the equilibrium between two unique conformational states of the molecular switch.

The switch that regulates the lac operon is arguably the most well characterized and therefore serves as the paradigm for understanding gene regulation (recently reviewed by Wilson et al (2007)) ${ }^{1}$. The switch is a two component system and consists of a repressor molecule and an operator. The repressor is a 360 amino acid protein that has a modular structure, composed of an $\mathrm{NH}_{2}$-terminal or "headpiece" domain ( 60 residues) and a $\mathrm{COOH}$-terminal "core" domain. The headpiece contains the classical helix-turn-helix (HTH) motif that recognizes and binds to an operator sequence while the "core" domain is responsible for inducer binding and contains

¥To whom correspondence should be addressed (lewis@mail.med.upenn.edu).

Publisher's Disclaimer: This is a PDF file of an unedited manuscript that has been accepted for publication. As a service to our customers we are providing this early version of the manuscript. The manuscript will undergo copyediting, typesetting, and review of the resulting proof before it is published in its final citable form. Please note that during the production process errors may be discovered which could affect the content, and all legal disclaimers that apply to the journal pertain. 
the dimerization interface. The second component of the switch is the operator, a short stretch of DNA that is pseudo palindromic. In the lac operon, the primary operator is positioned just upstream of the gene for $\beta$-galacotosidase ${ }^{2}$. As a negative regulator, the repressor associates with the operator sequence and physically blocks transcription of the genes that are necessary for lactose metabolism. The inducer molecule relieves repression by altering the repressoroperator equilibrium; stabilizing a conformation that is incompatible with operator binding ${ }^{3}$. The natural inducer of the lac operon is an analogue of lactose, allolactose, but a gratuitous inducer such as 1-isopropyl- $\beta$-D-thiogalactoside (IPTG) can also effectively decrease the binding affinity between the repressor and its operator. Since the inducer binds to a site that is distal to the DNA binding domain, the signal is transmitted through the molecule by altering the conformation of the repressor; this structural rearrangement of the repressor results in an allosteric transition.

Monod, Wyman and Changeaux (MWC) first described how structural changes could alter a protein's ability to perform a given function and coined the term allostery ${ }^{4}$. The MWC model assumes: (a) that allosteric proteins are oligomeric with at least one axis of symmetry; (b) these proteins adopt two distinct conformations (designated as R and T by MWC and described here as $\mathrm{R}$ and $\mathrm{R}^{*}$ ) (c) the conformational states have different affinities for the substrates. In this instance the $\mathrm{R}$ and $\mathrm{R}^{*}$ conformations have different affinities for the inducer and the operator; (d) each substrate (i.e. operator or inducer) binds to independent sites on each monomer; and finally (f) the molecular symmetry is conserved in the transition from one state to another. The lac repressor is an allosteric protein; it is oligomeric and each monomer has two well separated substrate binding sites, one for the operator and another for the inducer. Biochemical and structural studies have also demonstrated that the repressor adopts two distinct quaternary structures, which correspond to the induced and repressed states (see Wilson et al (2007)) ${ }^{1}$.

Here we describe a set of experiments that allowed us to elucidate the in vivo thermodynamic parameters that characterize the binding of inducer to the two conformational states of the repressor as well as the equilibrium constant between these two states. By measuring the effect of zero, one or two inducers binding to the repressor, we were able to extract equilibrium constants and develop a thermodynamic model to describe the allosteric process.

\section{Results}

\section{In vivo Induction Analysis}

Several mutant repressor molecules have been identified that bind to the operator DNA with wild type affinity but are incapable of induction ${ }^{5}$. These substitutions, classified by an $\mathrm{I}^{S}$ phenotype, are either defective in inducer binding or cannot transmit the allosteric signal to the DNA binding domain. The position of the $\mathrm{I}^{s}$ point mutations appear in five general locations with respect to the primary sequence, and include residues 70-80, 90-100, 190-200, 245-250 and 272-277. When the position of these mutations are mapped onto the protein structure, as is illustrated in Figure 1, they cluster at either the dimer interface or in close proximity to the inducer binding site. Presumably mutants within the ligand binding pocket interfere with the ability of the repressor to bind the inducer while mutants at the dimer interface disrupt the signaling process ${ }^{6}$. The crystal structure of the repressor bound to the inducer illustrates that IPTG forms hydrogen bonds to the amino acid side chain of Asp149, Arg197, Asn246 and Asp274, as well as van der Waals interactions with a hydrophobic surface created by Ile79, Leu148, Phe161, Asn291, Phe293 and Leu296 ${ }^{7}$. In fact, mutating any of these residues frequently results in the $\mathrm{I}^{s}$ phenotype ${ }^{5}$. However, as one would anticipate not all mutations in the inducer binding pocket affect induction equally. One particularly potent mutation in the ligand pocket, R197G, completely disrupts inducer binding but does not alter the repressor's ability to fold or bind to the operator ${ }^{8}$. The side chain of R197 anchors the galactose ring of the inducer by forming two hydrogen bonds with the $\mathrm{C} 2$ and $\mathrm{C} 3$ hydroxyls. To establish how 
inducer binding effects the allosteric properties of the repressor we used the R197G mutation and created heterodimeric repressors with zero, one or two functional inducer binding sites.

As described previously ${ }^{9}$, heterodimeric repressors were created by altering the C-terminal dimer interface of the wild type, dimeric lac repressor. These heterodimeric repressors have a unique monomer-monomer interface that allow us to probe the effect of asymmetric changes in the ligand pocket. The R197G mutation was incorporated into one or both monomers, producing heterodimeric repressors with zero, one or two non-functional inducer binding pockets (Table 1). Subsequently, the heterodimeric repressors were introduced into cells containing the GFPmut3.1 reporter, whose transcript is controlled by the binding of the repressor to a chimeric operator ${ }^{9}$. Cells transformed with plasmids containing the heterodimers were then grown in the presence and absence of $2.5 \mathrm{mM}$ IPTG and the levels of transcription were determined by measuring GFP fluorescence. As shown in Figure 2a, the heterodimeric repressors exhibit tight repression but disruption of the ligand binding sites alter the relative induction. The heterodimeric repressor with both sites intact has near full induction (76\% max expression) similar to both the dimeric and tetrameric form of the wild type lac repressors (Figure S1). When either one of the inducer binding sites is eliminated, induction is far from optimal and repression is only slightly relieved leading to less than $20 \%$ maximal induction. As expected, when both inducer binding pockets are destroyed repression can only be slightly relieved at the highest concentration of the inducer tested. Since this heterodimeric repressor binds to an asymmetric operator, we also explored if the orientation of the repressor containing a single functional ligand binding site with respect to the promoter affected induction. The asymmetric operator was inserted in both orientations into the reporter plasmid. The level of induction was identical irrespective of whether the inducible monomer was proximal or distal to the promoter (Figure 2a).

The induction measurements were repeated over a wide range of inducer concentrations $(0.1$ $\mathrm{nM}$ to $50 \mathrm{mM}$ ) to insure complete induction (Figure $2 \mathrm{~b}$ ). Again, when both inducer sites are functional we observe $80 \%$ of the maximal expression of GFP even at the highest concentration of inducer; the fluorescent signal never reaches the same level as that obtained when no repressor is present. This suggests that even when both inducer sites are fully occupied, there is still residual binding to the operator resulting in incomplete induction. Consistent results were also obtained with both the tetrameric and dimeric wild type repressors and the lack of full induction may represent the repressor-operator-inducer complex recently identified with small angle scattering ${ }^{10}$. Both of these wild type repressor constructs behaved similarly since the auxiliary operator sites required for increased repression by the tetrameric repressor are absent.

Induction was further limited by inactivating one of the binding sites. Repressors with one functional ligand binding site never exceed $20 \%$ of the maximum induction even at very high concentrations of the inducer. The titration curves suggest that although repression can be partially relieved upon binding a single inducer, two molecules of IPTG bound to the repressor are needed for complete induction, such that one is not enough. To ensure that the observed phenotype was not an artifact of the R197G mutation, additional constructs containing the R197A mutation were also tested. Both mutations demonstrated identical phenotypes (Figure S2) supporting the working hypothesis that mutating residue R197 affects inducer binding alone. By fitting the induction data to a 'two state' model we can establish the conformational equilibrium constant in the MWC model as well as the binding affinities of the inducer to the repressed and induced conformations.

Extracting the Thermodynamic Parameters-A fundamental tenant of the MWC model is that allosteric proteins exits in two conformations with different relative activities. In the case of the lac repressor, the two conformations are differentiated by their preferential 
binding to either the operator, $\mathrm{O}$, or inducer ligand, I. Here, we designate the conformation that prefers to bind DNA as the active form, R, and the conformation that prefers to bind inducer as the inactive, $\mathrm{R}^{*}$, form. The two conformation are in equilibrium (1), such that $K_{R^{*} R}=\left[R^{*}\right] /$ $[R]$ :

$$
R \stackrel{K_{R * R}}{\Longleftrightarrow} R *
$$

Both of these repressor conformations can bind to the operator in an equilibrium manner, but equilibrium values are not identical:

$$
\begin{gathered}
R+O \stackrel{K_{R O}}{\Rightarrow} R O \\
R *+O \stackrel{K_{R * O}}{\Rightarrow} R * O
\end{gathered}
$$

where $K_{R O}=[R O] /[R][O]$ and $K_{R^{*} O}=\left[R^{*} O\right] /\left[R^{*}\right][O]$ are the respective association constants. Although both conformations can bind to the operator, the ratio of equilibrium constants, $s=$ $K_{R^{*} O} / K_{R O}$, must be less than 1 for induction to occur.

In the classic MWC model, the inducer also binds to both conformations of the repressor with different affinities; it binds to the active $(\mathrm{R})$ conformation with an affinity given by the binding constant $\mathrm{K}_{\mathrm{IR}}=[\mathrm{RI}] /[\mathrm{R}][\mathrm{I}]$ and to the inactive $\left(\mathrm{R}^{*}\right)$ conformation with an affinity given by the binding constant $\mathrm{K}_{\mathrm{IR}}{ }^{*}=\left[\mathrm{R}^{*} \mathrm{I}\right] /\left[\mathrm{R}^{*}\right][\mathrm{I}]$. Since the functional unit of the repressor is dimeric, the repressor can bind two inducer molecules. The two binding sites are distant from one another and there is no direct interaction between inducers such that the inducer molecules bind independently to each of the binding pockets with identical affinities, i.e. there is no contribution to cooperativity from direct ligand-ligand interaction. This assumption is borne out by the observation that the dependence of the apparent rate of dissociation of the repressoroperator complex with respect to inducer concentration is noncooperative ${ }^{11}$. In accordance with the MWC model, inducer binding to the repressor drives the repressor equilibrium towards the inactive $\mathrm{R} *$ conformation and therefore, induction only requires that the inducer has a greater affinity for the inactive conformation $\mathrm{R}^{*}$ than the active conformation $\mathrm{R}$, i.e. $\mathrm{K}_{\mathrm{IR} *}>\mathrm{K}_{\mathrm{IR}}$.

The level of transcript produced in our GFP assay can be modeled using a standard ligand binding isotherm. The signal (E) is a function of the concentration of unbound repressor with respect to its operator dissociation constants (the repressor concentrations at which there would be $50 \%$ site occupancy).

$$
\frac{E}{E_{\max }}=\frac{1}{\left(1+\frac{\left[R_{\mathrm{TOT}}\right]}{\left[R_{50}\right]}\right)}
$$

In the absence of repressor, the fluorescent signal, $\mathrm{E}_{\max }$, is constitutively produced in this system. Since the repressor can adopt two distinct conformations, $\mathrm{R}$ and $\mathrm{R} *$, that have different operator binding dissociation constants then the binding isotherm takes the form: 


$$
\frac{E}{E_{\max }}=\frac{1}{\left(1+\frac{\left[R_{a}\right]}{\left[R_{50}\right]}+\frac{\left[R_{a}^{*}\right]}{\left[R_{50}^{*}\right]}\right)}
$$

The relative binding affinity is a function of the concentration of unbound repressor species in the two forms, $\left[\mathrm{R}_{\mathrm{a}}\right]$ and $\left[\mathrm{R}_{\mathrm{a}}\right]$ and their respective dissociation constants for operator binding $\left(\left[\mathrm{R}_{50}\right]=1 / \mathrm{K}_{\mathrm{RO}},\left[\mathrm{R}^{*}{ }_{50}\right]=1 / \mathrm{K}_{\mathrm{R} * \mathrm{O}}\right)$. As the concentration of the repressor in the active form increases the fractional expression decreases. Similarly, increasing the affinity of the repressor for its operator also decreases the fractional expression.

The fractional expression described in Eqn. (3) can be expressed as the product of two dimensionless ratios: the product of the ratio of the total concentration of the repressor to its operator binding dissociation constant in the active form, $\left[\mathrm{R}_{\mathrm{tot}}\right] /\left[\mathrm{R}_{50}\right]$, and the fractional amount of unbound repressor in the active form relative to the total concentration of repressor, $\left[\mathrm{R}_{\mathrm{a}}\right] /\left[\mathrm{R}_{\mathrm{tot}}\right]$. Similarly, $\left[R_{a}^{*}\right] /\left[\left[R_{\mathrm{tot}}\right]\right.$ is the fractional amount of unbound repressor in the induced form relative to the total concentration of repressor, such that Equation 3 takes the form:

$$
\frac{E}{E_{\max }}=\frac{1}{\left(1+\frac{\left[R_{\mathrm{tot}}\right]\left[R_{a}\right]}{\left[R_{50}\right]\left[R_{\mathrm{tot}}\right]}+\frac{\left[R_{\mathrm{tot}}\right]\left[R *_{a}\right]}{\left[R *_{50}\right]\left[R_{\mathrm{tot}}\right]}\right)}
$$

We defined a variable $f$ as $\left[\mathrm{R}_{\mathrm{a}}\right] /\left[\mathrm{R}_{\mathrm{tot}}\right]$, which depends upon both the inducer concentration and the conformational equilibrium constant and another variable $r$ as the ratio $\left[R_{t o t}\right] /\left[R_{50}\right]$. Since the amount of repressor species bound to operator is a small fraction of the unbound species, we can assume that $\mathrm{f}^{*}=1$ - $\mathrm{f}$ and the ratio of the operator affinities for the two repressor forms for operator is simply $\mathrm{K}_{\mathrm{R} * \mathrm{O}} / \mathrm{K}_{\mathrm{RO}}=\mathrm{s}$. This allows us to express the relative binding affinity in terms of three variables, $r$, $f$ and $s$, where $e=E / E_{\max }$.

$$
e=\frac{1}{(1+r(f+s(1-f)))}
$$

The linked equilibria that describes the binding of the repressor to its operator and to the inducer are illustrated in Figure 3. There are twelve different repressor species that contribute to the total repressor concentration.

$$
\begin{aligned}
& {\left[\mathrm{R}_{\mathrm{tot}}\right]=[\mathrm{R}]+2[\mathrm{RI}]+[\mathrm{R} *]+2[\mathrm{R} * \mathrm{I}]+\left\{\left[\mathrm{RI}_{2}\right]+\left[\mathrm{R} * \mathrm{I}_{2}\right]\right\}+} \\
& {[\mathrm{RO}]+2[\mathrm{RIO}]+\left\{\left[\mathrm{RI}_{2} \mathrm{O}\right]\right\}+[\mathrm{R} * \mathrm{O}]+2[\mathrm{R} * \mathrm{IO}]+\left\{\left[\mathrm{R} * \mathrm{I}_{2} \mathrm{O}\right]\right\}}
\end{aligned}
$$

When there are many more copies of the repressor than operators, the species bound to the operator ( $\mathrm{RO}, \mathrm{RIO}, \mathrm{RI}_{2} \mathrm{O}, \mathrm{R} * \mathrm{O}, \mathrm{R} * \mathrm{IO}$, and $\mathrm{R}^{*} \mathrm{I}_{2} \mathrm{O}$ in Eqn. 6) represent a small fraction of the total repressor concentration (an approximation of repressor and operator copy numbers were derived from plasmid construction, see methods) and can be neglected. This simplifies the subsequent equations and permits a closed form solution. The concentration of unbound repressor that exists in the $\mathrm{R}$ and $\mathrm{R}$ * conformations is the total of all unbound repressor species, $\left[\mathrm{R}_{\mathrm{a}}\right]$ and $[\mathrm{R} * \mathrm{a}]$ respectively: 
For the heterodimeric repressor with two inducer binding sites there are six unbound species in equilibrium, namely $\mathrm{R}, \mathrm{RI}, \mathrm{RI}_{2}, \mathrm{R} *, \mathrm{R} * \mathrm{I}$, and $\mathrm{R} * \mathrm{I}_{2}$ (the 2 -site case). When one inducer binding site is removed, the terms contained in \{\} in equations 6a-c disappear, and there are only four unbound species: R, RI, R*, and R*I (the 1-site case). In both instances, the amount of repressor $[R]$ that can bind to the operator depends upon the value of conformational equilibrium constant, $K_{R R^{*}}$, the inducer concentration [I] and relative inducer binding affinities to the $\mathrm{R}$ and $\mathrm{R}^{*}$ conformations. These three parameters are responsible for establishing the relative amounts of active and inactive repressor, $\mathrm{R}$ and $\mathrm{R}^{*}$, as described below. When the heterodimeric repressor has a single inducer binding pocket the amount of the various repressor/inducer species are governed by the linked equilibria on the left half of Figure 3 . The concentration of unbound repressor in the active and inactive forms is the sum of the first two terms in Eqns. 6a-b respectively, while the total repressor concentration is the sum of the first four terms in equation $6 \mathrm{c}$. When both ligand pockets are functional, the linked equilibria includes the right half of Figure 3 and the total repressor concentrations includes the \{\} bracket terms in equations $6 \mathrm{a}-\mathrm{c}$.

The concentration of all repressor species can be evaluated in terms of the concentration of one species, namely the free active repressor, $\left[R_{a}\right]$, using the appropriate equilibrium constants and the fraction of repressor in the unbound $\mathrm{R}$ form, $\mathrm{f}=\left[\mathrm{R}_{\mathrm{a}}\right] /\left[\mathrm{R}_{\mathrm{tot}}\right]$, which is given by the ratio of two binding polynomials. For the heterodimeric repressor that has two functional inducer binding pockets, the ratio of two binding polynomials is given by:

$$
f_{2}=\frac{\left[R_{a}\right]}{\left[R_{\mathrm{tot}}\right]}=\frac{\left(1+[I] K_{I R}\right)^{2}}{\left(1+[I] K_{I R}\right)^{2}+K_{R R_{*}}\left(1+[I] K_{I R *}\right)^{2}}
$$

We note that as in the original MWC model, the presence of factors having the form $(1+[\mathrm{L}]$ $\mathrm{K})^{\mathrm{n}}$ results from independent ligand binding at $\mathrm{n}$ identical sites, and it properly accounts for the statistical factor arising from the macroscopic indistinguishability of distinct microscopic species IR and RI etc. Similarly, the fraction of repressor in the R conformation when there is a single inducer binding site can be described by the ratio of corresponding polynomials

$$
f_{1}=\frac{\left[R_{a}\right]}{\left[R_{\mathrm{tot}}\right]}=\frac{\left(1+[I] K_{I R}\right)}{\left(1+[I] K_{I R}\right)+K_{R R *}\left(1+[I] K_{I R *}\right)}
$$

When both pockets are eliminated or in the absence of inducer, the fraction of the repressor in the $\mathrm{R}$ conformation reduces to:

$$
f_{0}=\frac{1}{1+K_{R R *}}
$$


At saturating concentration of inducer the fraction of free repressor depends only upon the conformational equilibrium, $\mathrm{K}_{\mathrm{RR}}$, and the relative binding of the inducer to the repressor in the $\mathrm{R}$ and $\mathrm{R} *$ conformations, as described by Eqns $8 \mathrm{a} \& \mathrm{~b}$.

$$
\begin{gathered}
f_{1}^{\prime}=\lim _{[I] \rightarrow \infty} f_{1}(I)=\frac{1}{1+K_{R R_{*}} \frac{K_{I R *}}{K_{I R}}} \\
f_{2}^{\prime}=\lim _{[I] \rightarrow \infty} f_{2}(I)=\frac{1}{1+K_{R R_{*}}\left\{\frac{K_{I R_{*}}}{K_{I R}}\right\}^{2}}
\end{gathered}
$$

By substituting Eqns. 7-8 into Equation 5 we can relate the relative amount of transcript produced at limiting conditions to four parameters: (a) the ratio of total repressor concentration to the active form operator binding dissociation constant, $r$, (b) the ratio of inducer binding to the inactive and active repressor conformations, $\mathrm{x}=\frac{K_{I R^{*}}}{K_{I R}}$, (c) the equilibrium between these two different conformations, $\mathrm{K}_{\mathrm{RR}}$, and (d) the ratio of operator affinities of the two different repressor forms, $\mathrm{s}$.

$$
e_{n}=1 /\left(1+r\left(1 /\left(1+K_{R R *} x^{n}\right)\right)+r s\left(1-1 /\left(1+K_{R R *} x^{n}\right)\right)\right)
$$

The three values of $e_{i}$, for $n=0,1$, or 2 are the experimentally observed levels of GFP expression with zero inducer (or with zero sites), at high inducer concentration for the 1-site repressor and high inducer concentration for the 2-site repressor, respectively. Rearranging Eqn. 9 we define the variable $y_{i}$ as :

$$
y_{n}=1 / e_{n}-1=r\left(1 / /_{\left(1+K_{R R *} x^{n}\right)}+s\left(1-1 / /_{\left(1+K_{R R *} \cdot x^{n}\right)}\right)\right)
$$

By evaluating the ratio of the 1 and 2 site cases to the zero inducer case, we can eliminate the variable $r$, and obtain two equations in three variables $\mathrm{s}, \mathrm{x}$ and $\mathrm{K}_{\mathrm{RR}}$ *

$$
y_{n} / y_{o}=\left.\left({ }_{\left(1+K_{R R *} x^{n}\right)}+s\left(1-1_{\left(1+K_{R R *} x^{n}\right)}\right)\right)\right|_{\left(1 / 1_{\left(1+K_{R R *}\right)}+s\left(1-1 /{ }_{\left(1+K_{R R *}\right)}\right)\right)}
$$

where $n=1$ or 2 .

The two parameters, $\mathrm{x}$ and $\mathrm{K}_{\mathrm{RR}} *$ were first fit to the experimentally observed values, $\mathrm{y}_{1}$ and $\mathrm{y}_{2}$, on the left-hand side of Eqn. 11 by a straightforward grid search assuming there is no binding of the inactive repressor $\left(\mathrm{R}^{*}\right)$ to the operator $(\mathrm{O})$, i.e. $\mathrm{s}=0$. We then solved for $\mathrm{r}$ using Eqn. 10. 
Subsequently, the absolute value of the inducer binding constant, $\mathrm{K}_{\mathrm{IR}}$, was determined by fitting the expression level curves vs. inducer concentration for both the 1-site and 2-site dimers using the parameter values $\mathrm{x}, \mathrm{r}$ and $\mathrm{K}_{\mathrm{RR}^{*}}$. The value of $\mathrm{K}_{\mathrm{IR}} *\left(=1 / \mathrm{K}_{\mathrm{d}(\mathrm{IR} *)}\right)$ was refined by bisection to minimize the mean absolute error in fractional expression. The other inducer binding constant was then determined as $\mathrm{K}_{\mathrm{IR}}=\mathrm{xK}_{\mathrm{IR}} *$.

\section{Analysis of the Extracted Parameters}

The value of $K_{R R} *$ was determined to be $2 \pm 0.5$, which is in good agreement with previous in vitro measurements ${ }^{12}$ and consistent with our observation that the repressor prefers to crystallize in the induced $\mathrm{R}^{*}$ conformation in the absence of the ligand, regardless of the crystallization conditions ${ }^{7}$. The ratio of the inducer binding constants, $x=K_{I R} / K_{I R}=15 \pm 3$ illustrates that the inducer preferentially binds to the $\mathrm{R}^{*}$ conformation. The in vivo ratio of equilibrium constants is, however, somewhat less than that observed by O'Gorman et al. in vitro. ${ }^{12}$ The calculated dissociation constant for active repressor-inducer binding, $\mathrm{K}_{\mathrm{d}(\mathrm{IR})}$, is 4 $\pm 2 \mathrm{uM}$. Since the ratio of the two binding constants, $\mathrm{K}_{\mathrm{IR}} * / \mathrm{K}_{\mathrm{ir}}$, is $\sim 15$, we can infer that the corresponding dissociation constant for the inducer to the inactive repressor, $\mathrm{K}_{\mathrm{d}(\mathrm{IR} *)}$, is $15 * 4 \mathrm{uM}$ or $60 \mathrm{uM}$. Overall, the values of $\mathrm{K}_{\mathrm{RR}} *$ and $\mathrm{x}$ are consistent with our structural observation that the conformation of the apo repressor and the repressor-IPTG complex are essentially isomorphous ${ }^{7}$.

The ratio of the total repressor concentration to its operator dissociation constant, $\left[R_{t o t}\right] /\left[R_{50}\right]$ is a complex quantity that reflects the binding affinity of the repressor for the operator and the concentrations of the repressor and operator in the cell. We find that this ratio is $150 \pm 50$. This implies that there is a vast excess of repressor compared to that required for $50 \%$ operator binding, which is consistent with our in vivo assay system (see methods). The model also predicts that the switch is leaky. In the absence of inducer, only a fraction, $\mathrm{f}_{0}=1 /\left(1+\mathrm{K}_{\mathrm{RR}} *\right)$, of the repressor molecules adopt the $\mathrm{R}$ conformation, leading to a net active repressor concentration of $\mathrm{f}_{0}=1 /(1+2)$ that is 'only' $\sim 50$-fold above the repressor-operator dissociation constant. Although the repressor is operating under saturating conditions and the vast majority of the operators are bound by the repressor, a small amount of transcript, roughly 2-3\%, would be produced since, $E / E_{\max }=1 /(1+50)$. In the absence of inducer, a detectable level of background expression is observed, consistent with the values calculated from the model.

According to the MWC model, the contribution the inactive conformation makes to the binding of the repressor to the operator is negligible. The previously discussed parameters were determined under this assumption ( $\mathrm{s}=0$ ); however, to test its validity, we repeated the above fitting procedure allowing the induced conformation $\mathrm{R}^{*}$ to bind $\mathrm{O}$ with a range of affinities ( 0 $\leq \mathrm{s} \leq 1$ ). The resulting parameters are tabulated in Table 2 . When the $\mathrm{R}^{*}$ conformation binds the operator with an affinity of 1/10000th or less than the active form $R$, the model produces very similar quality fits to the limiting expression and IPTG titration curves, with almost identical output parameters. Assuming a somewhat higher relative affinity of 1/1000th produces a slightly poorer fit to the limiting expression data, but an equally good fit to the whole induction curve. At a higher relative affinity of $s=1 / 100$ th, the fit is poor. Essentially, when the relative affinity of $\mathrm{R}^{*}$ for $\mathrm{O}$ binding is large, the inducer cannot pull the repressor off the operator and as a consequence the system is not inducible. Overall, the best fit parameters are slightly different than when we assume $s=0$, notably the conformational equilibrium is somewhat closer to unity, but the differences fall within the estimated uncertainty ranges and the same qualitative picture emerges: There is a small but significant preference for the inactive form of repressor in the absence of inducer, a large excess of repressor relative to its $\mathrm{K}_{\mathrm{d}}$ for operator binding and an order of magnitude tighter binding of inducer to the inactive repressor form. We therefore conclude that the s value must be on the order of 1/1000th or less to explain the in vivo data. This allows us to effectively ignore $\mathrm{R} *$ binding to operator. 
The quality of the model and the accuracy of the parameters can be assessed by comparing the calculated and observed induction curves (Figure 4). The experimental data fits the calculated model very well yielding a mean unsigned error of $2 \%$. The best fit model slightly overestimates the slope of expression vs. I at the midpoint. This could result from systematic overestimation of the effective IPTG activity in vivo, i.e. the actual activity of IPTG is somewhat lower than the nominal concentration values used in plotting the data. Another measure of the validity of the derived constants was assessed by determining the ratio of the $[\mathrm{I}]_{50}$ values for the 1 -site to 2 -site cases. A calculated value of 2.5 agrees well with the experimentally observed value of $\approx 3$. The agreement between the observed and calculated ratios is a good measure of selfconsistency since this ratio is not an adjustable parameter. In the fitting to the model, (although not in the model itself), we assumed that the concentration of repressor was much greater than the operator such that the amount of repressor bound to operator is small compared to the total and free amounts of repressor. Estimates of the repressor copy number are imprecise, but the ratio of repressor to operator in a similar system was estimated to be $\sim 100: 1^{13}$. This ratio is sufficiently large such that the fractional error that results from neglecting the concentration of the bound repressor species in Equation $2 \mathrm{c}$ is $1 / 100$, less than a few percent. This permits us to obtain closed form expressions for limiting expression levels, and a straightforward physical interpretation in terms of the basic model parameters with little numerical error. A more exact model may be applied for low repressor copy number by keeping all the terms in equation $2 \mathrm{c}$, and fitting iteratively, assuming that the total repressor remains constant at each step.

The expression of GFP in the reporter system depends upon the inducer concentration and its relative affinity for the $\mathrm{R}^{*}$ and $\mathrm{R}$ conformations of the repressor, which we estimate to be $4 \mathrm{uM}$ and $60 \mathrm{uM}$ respectively. This difference in binding affinities results in curves that are slightly sigmoidal when expression/repression values are plotted against inducer concentration. The mid-point, somewhere between the two individual dissociation constant values, is $\sim 25 \mathrm{uM}$ and the plot has a Hill coefficient of $\sim 1.25$ (Figure S3) is consistent with numerous studies ${ }^{14 ;}$ 15; 16. This mild cooperativity arises from the inducer promoted shift of repressor conformational equilibrium towards the higher affinity form. However, since these curves are all characterized by a single inflection point, without further independent data only a single 'apparent' inducer affinity constant is extractable.

In the above model of heterodimeric repressor action, we have assumed that the binding pocket mutations R197G/A in one of the monomers acts by blocking binding of inducer to one site, and that this is responsible for the drop in maximal expression from $75 \%$ to $18 \%$. Inducibility of the repressor would also be reduced if the mutation caused the conformational equilibrium to shift towards the $\mathrm{R}$ form (i.e. $\mathrm{K}_{\mathrm{RR}} *$ decreases). While it is unlikely that a binding pocket mutation would exert its effect through $\mathrm{K}_{\mathrm{RR}} *$ rather than $\mathrm{K}_{\mathrm{IR}}$ and $\mathrm{K}_{\mathrm{IR}} *$ we can consider this possibility in our model. Using the two binding site Eqn $5 \mathrm{a}$ rather than $5 \mathrm{~b}$ to fit the "1-site mutation' induction curve and adjusting only $\mathrm{K}_{\mathrm{RR}}$, we can model a reduced maximal expression level of $18 \%$ by setting $\mathrm{K}_{\mathrm{RR}} *=0.15$. In this model, the heterodimeric repressor with one site R197G/A mutation still binds two inducers with unchanged affinity, but the mutation has pushed the repressor further into the active conformation. However this model predicts that at zero inducer there is no detectable expression, i.e. the switch is not leaky, which is inconsistent with the experimental data.

\section{Discussion}

\section{A Thermodynamic Perspective}

The lac repressor is a model system for exploring gene regulation and the mechanism by which effector molecules regulate transcription. Measuring induction using the hetero-dimeric repressors with zero, one and two inducer pockets allowed us to unambiguously extract three 
important thermodynamic quantities: the equilibrium constant between the induced and repressed conformations of the repressor, $\mathrm{K}_{\mathrm{RR}^{*}}$, the relative affinities of each conformation for inducer $\left(\mathrm{K}_{\mathrm{IR}} / \mathrm{K}_{\mathrm{IR}}\right)$, and the amount of repressor in the cell relative to its operator binding dissociation constant. In addition, determining these parameters allows us to begin exploring how each aspect of the repressor contributes to its functionality by modeling theoretical changes in each parameter.

From modeling the in vivo data it appears that effective repression in the absence of inducer ( $<4 \%$ expression) requires a large excess of active repressor with respect to its operator binding constant (50-fold). While a background expression of $4 \%$ may be less than ideal, given the other thermodynamic parameters of the repressor, an $r$ value of 150 appears to be optimal. To better understand the relationship between the ratio of active repressor concentration and operator affinity, a series of simulated induction curves were generated by varying the $\left[\mathrm{R}_{\mathrm{TOT}}\right] /\left[\mathrm{R}_{50}\right]$ ratio (Figure 5a). By increasing the ratio to 500 or 1000 , which can be achieved by either increasing the active repressor concentration or increasing the repressor-operator affinity, constitutive expression can be minimized to less than $1 \%$. However, increases in the ratio also affect the dynamic range of the switch. Increased repression comes at the expense of induction and the inducer cannot relieve repression to more than 50\% max expression. Conversely, if the ratio is too small, then the switch can be induced but background expression is very high. When the ratio of $r=150$ then the background expression appears to be minimized while balancing maximal induction near $75 \%$. Experimentally, both the 1-site and 2-site inducer binding cases show a plateau in expression level at transcriptional levels less than 100\% suggesting that there is always a small fraction of the repressor that can adopt the active (R) conformation and block transcription.

The equilibrium distribution of repressors in both the active and inactive conformation is an additional parameter which controls the repression and induction profile of the repressor. From modeling the experimental data, there appears to be a rather small energy difference between the two conformations of the repressor ( $\mathrm{R}$ and $\left.\mathrm{R}^{*}\right), \Delta G=k \operatorname{Tln}\left(K_{R R^{*}}\right)=0.4 \mathrm{kcal} / \mathrm{mole}$. This modest difference in energy allows the repressor to easily switch between the active and inactive states. Again we simulated induction curves by altering the equilibrium constant (designated as $\mathrm{L}$ according to the MWC nomenclature) to explore the impact of this parameter on repressor activity (Figure 5b). Since the active conformation of the repressor has preferential binding to the operator, when the apparent equilibrium constant is less than 1, and the active conformation of the repressor is dominant; repression is strong and there is minimal background expression. However, altering $\mathrm{K}_{\mathrm{RR}} *$ to increase the concentration of active repressor also increases the ratio $r$ discussed previously. This limits the dynamic range of expression and permits only a fraction of the possible induction. In contrast, when the equilibrium constant is large, the majority of the repressor is in the $\mathrm{R}^{*}$ conformation resulting in a switch that is leaky but fully inducible. At the fixed values for the other thermodynamic properties, the dynamic range of the switch is optimal when $L\left(K_{R R^{*}}\right)$ is between 1 and 10 . There is a low level of basal expression in the absence of inducer and nearly full induction at high concentrations of inducer.

Since repressor activity is a product of linked equilibria, the distribution of active and inactive repressors can be altered by adding either operator or inducer ligands. The system is repressed when there is an excess of active repressor with respect to its operator dissociation constant. Induction, on the other hand, requires an excess of inactive repressor. The inducer relieves repression by shifting the apparent conformational equilibrium, decreasing the concentration of active repressor. A single inducer binds 15 times more tightly to the $\mathrm{R} *$ conformation than the $\mathrm{R}$ conformation, which in energetic terms is about $1.6 \mathrm{kcal} / \mathrm{mole}$. In the presence of a single bound inducer, the apparent equilibrium constant increases, $K_{R R_{*}^{\prime}}=\exp ^{(0.4+1.6) / k Y} \approx 30$, stabilizing the $\mathrm{R}^{*}$ conformation; the $\left[\mathrm{R}_{\mathrm{a}}\right] /\left[\mathrm{R}_{50}\right]$ ratio drops to $\sim 5$-fold $(150 / 30)$. The additional 
energy shifts the apparent equilibrium further towards the $\mathrm{R}^{*}$ conformation thereby reducing the effective concentration of the repressor in the R conformation. Since there is less active repressor, polymerase has a greater opportunity to transcribe its message. Therefore, in the presence of high concentrations of inducer, the single site repressor can achieve a fractional induction level of $1 /(1+5)=16 \%$, which is consistent with our experimental observation. A second inducer molecule shifts the apparent equilibrium more dramatically. An additional 1.6 $\mathrm{kcal} / \mathrm{mol}$ of binding energy increases $K_{R R} *$ to $\sim 450$, driving the repressor further toward the $\mathrm{R}^{*}$ conformation and lowering the $\left[\mathrm{R}_{\mathrm{a}}\right] /\left[\mathrm{R}_{50}\right]$ ratio to less than unity $\sim 0.4$. As a consequence, in the presence of high levels of inducer we expect $1 /(1+0.33)=75 \%$ induction, which is very close to the experimental observation. Since the calculated energetic effects appear to be purely additive, we would suspect that cooperativity to be of little importance when it comes to induction. In fact, the Hill coefficient (at inducer the mid-point) is a rather modest 1.25 and conclude that cooperativity is not an essential requirement of the switch. Cooperativity is important for shifting the window in which an effector can elicit a response to some relatively narrow range of non-zero concentrations. For example, the cooperativity seen with hemoglobin is probably due to the fact that the partial pressures of $\mathrm{O}_{2}$ are never zero, and may only range from $40-80 \mathrm{mmHg}$. In contrast, in noncooperative (Langmuir isotherm type) binding, the rate of increase is greatest starting from zero effector, which works better as a switch in the lac system given the actual inducer concentration range.

In the previous examples, the experimentally determined value of $x=15$ was used to model induction as a consequence of altering the other parameters. Since the repressors response to inducer is dependent upon the ratio of relative affinities of the inducer for each conformation of the repressor, simulated induction curves were also produced with variations in $\mathrm{x}$ (Figure $5 c)$. For $x=1$, the inducer binds to both the active and inactive conformations with the same affinity and expression levels become insensitive to inducer. When the ratio is less than unity, the inducer binds more tightly to the active conformation, and actually decreases induction. The inducer ligand would then function more like a co-repressor. Only when the ratio is significantly greater than one does the system work as a true switch; having full repression at low inducer concentrations and complete induction at high concentrations of inducer. Unlike the other parameters which alter both repression and induction, no such balance exists for the value of $\mathrm{x}$ and as $\mathrm{x}$ increases to greater values, the switch functions even better. The measurements and analysis described were made using the gratuitous inducer, IPTG, it would be interesting to see if the value for $\mathrm{x}$, and thus the maximal expression level, is higher when the natural inducer, allolactose, is utilized. Regardless, this analysis suggests that induction of the lac repressor with IPTG can be improved by finding mutations which increase the ratio of binding affinities beyond 15 .

As the MWC model suggests, the repressor adopts two distinct conformations but only one of the conformations is functional. Therefore, the switch also depends on the relative operator binding of the two repressor conformations. To explore the importance of this parameter on repressor activity, we examined how variations in $\mathrm{s}$ alter the induction curves (Figure 5d). When the value of $\mathrm{s}$ is greater than unity, the inactive conformation of the repressor binds to the operator and the inducer again behaves as a co-repressor by increasing repression. At unity, both conformations have the same affinity for the operator and inducer has no affect. The switch will function only when the value of $\mathrm{s} \ll 1$. As $\mathrm{s}$ decreases there is greater induction which quickly approaches the limit as $s=0$. Based upon these curves, when $\mathrm{s}$ is less than 0.0005 , the system functions as if $\mathrm{s}=0$.

\section{A Kinetic Perspective}

The thermodynamic description of the switch is extremely useful for understanding the molecular mechanism of gene regulation. However, to fully appreciate how this switch 
functions, we must consider its activities in the context of its kinetic properties. The binding of the lac repressor to its operator has been well studied and all of the data are consistent with a bimolecular reaction (Eqn 10) having an association constant, $\mathrm{K}_{\mathrm{RO}}$ of $1.5 \times 10^{10} \mathrm{M}^{-1}$ under standard conditions ${ }^{11 ; 15}$. The equilibrium constant, of course, is the ratio of the association and disassociation rate constants:

$$
\mathrm{K}_{\mathrm{RO}}=[\mathrm{RO}] /[\mathrm{R}][\mathrm{O}]=\mathrm{k}_{\mathrm{a}} / \mathrm{k}_{\mathrm{d}}
$$

The dissociation constant has been measured experimentally for the wild type repressor and its natural operator, as $\mathrm{k}_{\mathrm{d}}=6 \times 10^{-4} \mathrm{~s}^{-1}$; using the above value of $\mathrm{K}_{\mathrm{RO}}$ this gives $\mathrm{k}_{\mathrm{a}}=7.5 \times 10^{6}$ $\mathrm{M}^{-1} \mathrm{~s}^{-1} 15 ; 17 ; 18$. A considerably greater association rate has been measured directly by Riggs et al. who found a value of $7 \times 10^{9} \mathrm{M}^{-1} \mathrm{~s}^{-1} 15$. In general, the three order of magnitude decrease in affinity that results from inducer binding can arise either from a decrease the on rate, an increase the off rate, or any combination of the two. Changes in the off rate have been estimated to range from 6-18 fold at concentrations of $10 \mathrm{uM}$ inducer, well above the inducer mid-point $15 ; 16$. This increase, while significant, implies that a large portion of the affinity loss is due to a decrease in the on rate. We therefore deduce that the inducer stabilizes the $\mathrm{R}^{*}$ conformation of the repressor by decreasing the on-rate by conservatively 10 -fold and, arguably by 100 -fold or more. It is instructive to speculate how altering these kinetic parameters can affect gene regulation and whether modulation of affinity through the association rate rather than the dissociation rate has any functional significance.

On average the fraction of the operator that is free of repressor is $f_{o}=1 /\left(1+[R] K_{R O}\right)$ for a given repressor concentration $[R]$. Since operator binding is actually a stochastic process, at any instance the operator is either occupied or not and there will be a distribution of occupation times. From the theory of stochastic processes ${ }^{19}$ the time that the operator is occupied by repressor is exponentially distributed with a mean of $\tau_{\mathrm{on}}=1 / \mathrm{k}_{\mathrm{d}}$. Similarly, the mean time the operator is free of repressor also varies exponentially, with a mean of $\tau_{\mathrm{off}}=1 / \mathrm{k}_{\mathrm{a}}[\mathrm{R}]$. Based upon the data of Riggs et al ${ }^{15}$, we can estimate that in the absence of inducer the mean occupancy time is $\sim 1600 \mathrm{sec}$. With an estimated repressor concentration of $20 \mathrm{nM}$ (O'Gorman) the mean time the operator is free of repressor in the absence of inducer is about $6 \mathrm{sec}$ using the lower estimate of $\mathrm{Ka}$, and $7 \mathrm{msec}$ using the upper estimate.

Transcription initiation takes a finite amount of time. Consequently, the fraction of time the operator is free and the duration of time it is free are both relevant for effective transcription. We hypothesize that the promoter must be free of repressor for long enough that the transcriptional machinery can progress to the point where it is not affected by repressor binding. The kinetics of transcriptional initiation at the lac promoter has been well studied. ${ }^{20}$ RNA polymerase binds to the promoter region forming a 'closed' complex, which then undergoes an 'isomerization' to form the open (strand separated) initiating complex. Assuming the isomerization step, which has a first order rate constant of $1.6 \times 10^{-2} \mathrm{~s}^{-1}$, approximates the time to initiate transcription, then the promoter must be free from the repressor for roughly 60 secs. This is considerably longer than the mean time free under high repression conditions. Now if the effect of the inducer were solely on the dissociation rate of the repressor, the mean occupancy time would be significantly reduced; but the mean time the operator were free of repressor would be unchanged, and therefore on average the time frame is still be too short for transcription to initiate. If the inducer affected repressor affinity by depressing the on rate, then the average time the promoter is free of repressor would increase allowing transcription to initiate. 


\section{Conclusion}

Elucidating the thermodynamic properties of the molecular switch is essential for developing a complete understanding of gene regulation. The MWC model for the allosteric transition applies beautifully to the lac molecular switch and accounts for the conformations of the repressor observed in the crystal structures. Here we have experimentally determined the equilibrium constants that account for the observed properties of the molecular switch. Our analysis suggests that the transition from the induced to the repressed conformation requires a relatively small amount of energy $(0.4 \mathrm{kcal} / \mathrm{mole})$ and only subtly favors the induced conformation. This soft equilibrium allows the activity of the repressor to be modified by adjusting the conformational equilibrium through the mechanisms of linked equilibria. Since both the inducer and operator preferentially bind to different conformations, binding of inducer provides energy to compete with operator binding. By increasing the effective concentration of the inactive repressor, inducer binding reduces the number of repressors in the active conformation and therefore reduces repression. Our analysis demonstrates that a single inducer provides enough energy to increase expression levels to $20 \%$ and that two inducers are required for maximal induction. In addition, from altering various parameters of the repressor and monitoring the affect on repressor activity, we have gained valuable insight into designing improved switches. Tuning the allosteric properties of the repressor will allow us to create better and novel molecular switches.

\section{Material and Methods}

\section{Repressor Plasmid Construction}

Construction of the dimeric wild type repressor plasmid (pBD21004), the heterodimeric plasmid (pBD22010) and the plasmids containing each of the constituent monomers (pBD21008 and pBD21903) have been described previously ${ }^{9}$. To introduce the R197A and R197G mutations into the pBD21004 and pBD21008 plasmids, full circle PCR mutagenesis was used with primers BD030 (5' GCT CTG GCT GGC TGG CAT AAA TAT C 3'), BD031 (5' CAG ACG CGC CGA GAC AGA AC 3') and primers BD032 (5' GGT CTG GCT GGC TGG CAT AAA TAT C 3'), BD031, respectively. After introducing the point mutations into each of these repressor genes, the two monomeric repressor genes were reintroduced into the heterodimeric plasmid. In short, the pBD21903 plasmid was digested with DrdI and the repressor gene was isolated via gel purification. Subsequently, the vector containing the recently mutated R197A or R197G gene (pBD21012, pBD21016) was digested with DrdI and purified. These vectors (also containing the Y282S monomeric mutation) were then ligated with the insert containing the complimentary repressor gene creating plasmids pBD22011 and pBD22012. The repressor plasmid is derived from the pACYC vector with a copy number around 15. Expression of lacI is under the control of the constitutive lac promoter. Estimates of repressor copy number are comparable with Oehler et $\mathrm{al}^{13}$.

\section{Reporter Plasmid Construction}

Construction of the reporter plasmid with GFPmut3.1 under the control of the lac promoter/ operator has been described previously ${ }^{9}$. In short, GFPmut 3.1 was introduced into the pBR322 vector and placed under control of the lac promoter/operator. To introduce operator changes producing chimeric operators, full circle PCR mutagenesis was used (operator 212:411 primers BD024 and BD021, operator 411:212 - primers BD020 and BD025). To explore the role of directionality on induction with repressor mutants containing asymmetric ligand pocket knockouts, two reporters were created and utilized. Each of these reporters contained a chimeric operator sequence with the orientation of the half sites inverted with respect to each other. In assays using the wild type (tetrameric or dimeric) repressor, the reporter containing the natural operator was used. 


\section{In Vivo Repression/Induction Assay}

To analyze the phenotypes of various repressor - operator combinations an in vivo fluorescent assay was used. To quantify the level of fluorescence and therefore indirectly measure the degree of transcription, cells were grown and analyzed in a Perkin Elmer Victor3 plate reader. In short combinations of repressors and operators were transformed and colonies were selected in triplicate for overnight culture growth. In addition, cells controlling the reporter only were also chosen to establish the level of maximal expression under non repressing conditions. For induction analysis, samples were grown in the absence of IPTG as well as in the presence of various amounts of inducer. Once samples reached saturation, $200 \mathrm{ul}$ aliquots were taken and introduced into flat bottom 96 well plates. A dilution plate was also prepared so that the optical density of the cultures could more accurately be determined. Each of these plates was then measured for GFPmut3.1 fluorescence (495nm excitation wavelength, $510 \mathrm{~nm}$ emission wavelength) and optical density (A590) on a Perkin Elmer Victor3 Plate reader. Fluorescence data was then processed to remove background fluorescence and normalize the GFP signal to the cell count. The normalized signals for biological replicates were then averaged to provide a relative fluorescent signal for each sample. Errors for each sample were determined from the standard deviation of the biological replicates. Fractional expression levels were calculated by dividing the average GFP signal for the sample with a given repressor by the signal of the sample containing no functional repressor.

\section{Supplementary Material}

Refer to Web version on PubMed Central for supplementary material.

\section{References}

1. Wilson CJ, Zhan H, Swint-Kruse L, Matthews KS. The lactose repressor system: paradigms for regulation, allosteric behavior and protein folding. Cellular \& Molecular Life Sciences 2007;64:3-16. [PubMed: 17103112]

2. Jacob F, Monod J. Genetic Regulatory Mechanisms in the Synthesis of Proteins. J Mol Biol 1961;3:318-356. [PubMed: 13718526]

3. Barkley, MD.; Bourgeois, S. Repressor Recognition of Operator and Effectors. In: Miller, JH.; Reznikoff, WS., editors. The Operon. Vol. Second. Cold Spring Harbor Laboratory; Cold Spring Harbor, NY: 1980. p. 177-220.

4. Monod J, Wyman J, Changeux JP. On the Nature of Allosteric Transitions: A Plausible Model. J Mol Biol 1965;12:88-118. [PubMed: 14343300]

5. Markiewicz P, Kleina LG, Cruz C, Ehret S, Miller JH. Genetic studies of the lac repressor. XIV. Analysis of 4000 altered Escherichia coli lac repressors reveals essential and non-essential residues, as well as "spacers" which do not require a specific sequence. Journal of Molecular Biology 1994;240:421-33. [PubMed: 8046748]

6. Pace HC, Kercher MA, Lu P, Markiewicz P, Miller JH, Chang G, Lewis M. Lac repressor genetic map in real space. Trends in Biochemical Sciences 1997;22:334-9. [PubMed: 9301333]

7. Daber R, Stayrook S, Rosenberg A, Lewis M. Structural Analysis of Lac Repressor Bound to Allosteric Effectors. Journal of Molecular Biology 2007;370:609-619. [PubMed: 17543986]

8. Spotts RO, Chakerian AE, Matthews KS. Arginine 197 of lac repressor contributes significant energy to inducer binding. Confirmation of homology to periplasmic sugar binding proteins. J Biol Chem 1991;266:22998-3002. [PubMed: 1744095]

9. Daber R, Lewis M. A Novel Molecular Switch. J Mol Biol. 2009

10. Taraban M, Zhan H, Whitten AE, Langley DB, Matthews KS, Swint-Kruse L, Trewhella J. Ligandinduced conformational changes and conformational dynamics in the solution structure of the lactose repressor protein. J Mol Biol 2008;376:466-81. [PubMed: 18164724]

11. Barkley MD, Riggs AD, Jobe A, Burgeois S. Interaction of effecting ligands with lac repressor and repressor-operator complex. Biochemistry 1975;14:1700-12. [PubMed: 235964] 
12. O'Gorman RB, Rosenberg JM, Kallai OB, Dickerson RE, Itakura K, Riggs AD, Matthews KS. Equilibrium binding of inducer to lac repressor.operator DNA complex. Journal of Biological Chemistry 1980;255:10107-14. [PubMed: 7000771]

13. Oehler S, Amouyal M, Kolkhof P, von Wilcken-Bergmann B, Muller-Hill B. Quality and position of the three lac operators of E. coli define efficiency of repression. EMBO Journal 1994;13:3348-55. [PubMed: 8045263]

14. Daly TJ, Matthews KS. Allosteric Regulation of Inducer and Operator Binding to the Lactose Repressor. Biochemistry 1986;25:5479-5484. [PubMed: 3535880]

15. Riggs AD, Bourgeois S, Cohn M. The lac repressor-operator interaction. 3. Kinetic studies. Journal of Molecular Biology 1970;53:401-17. [PubMed: 4924006]

16. Swint-Kruse L, Zhan H, Matthews KS. Integrated insights from simulation, experiment, and mutational analysis yield new details of LacI function. Biochemistry 2005;44:11201-13. [PubMed: 16101304]

17. Riggs AD, Newby RF, Bourgeois S. lac repressor--operator interaction. II. Effect of galactosides and other ligands. Journal of Molecular Biology 1970;51:303-14. [PubMed: 4320936]

18. Riggs AD, Suzuki H, Bourgeoss S. Lac repressor-operator interaction. I. Equilibrium studies. Journal of Molecular Biology 1970;48:67-83. [PubMed: 4915295]

19. Kampen, NGv. Stochastic processes in physics and chemistry. Vol. Rev and enl. North-Holland personal library; North-Holland, Amsterdam; New York: 1992.

20. Schlax PJ, Capp MW, Record MT Jr. Inhibition of transcription initiation by lac repressor. Journal of Molecular Biology 1995;245:331-50. [PubMed: 7837267] 


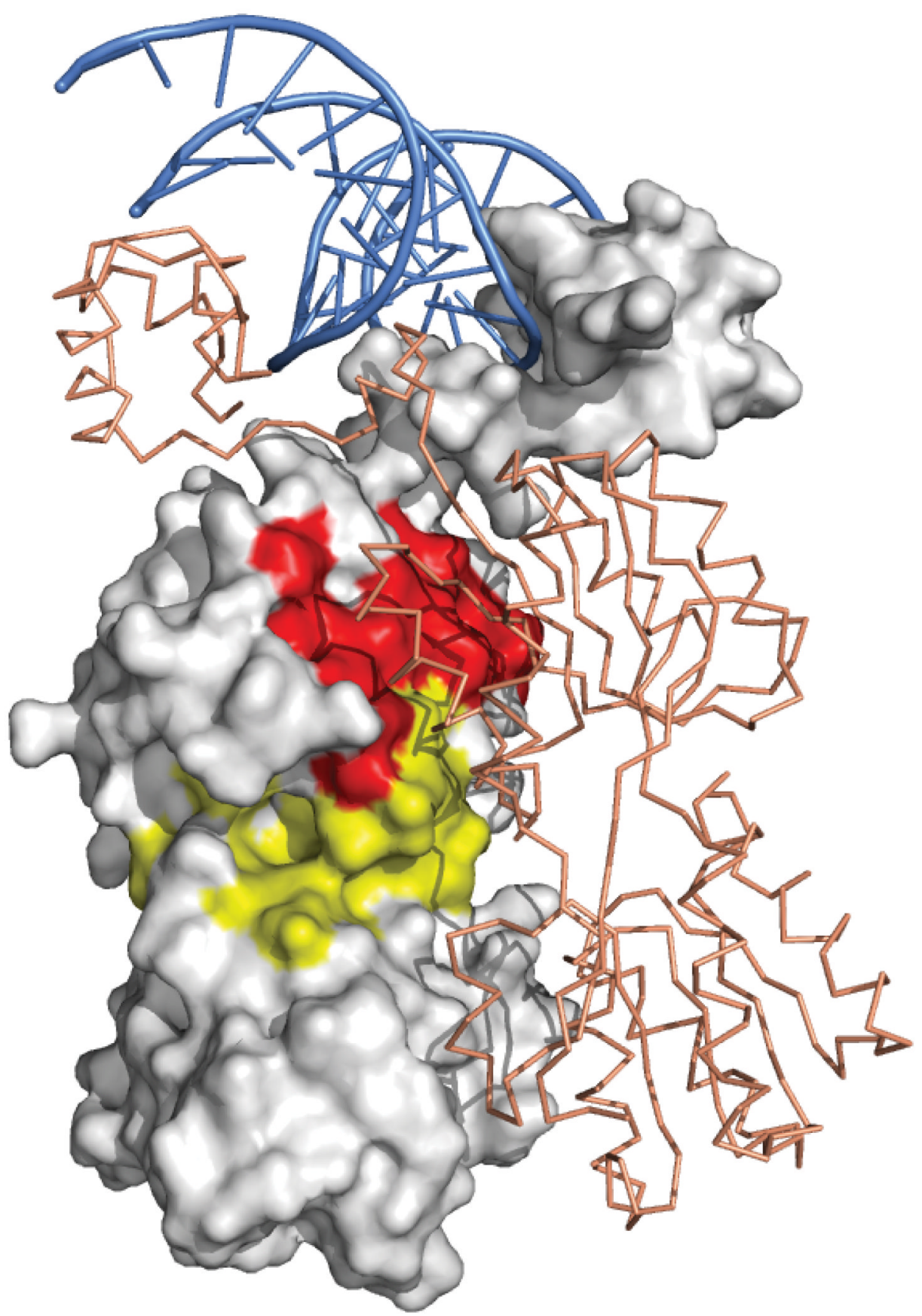

Figure 1.

The surface of the dimeric repressor depicting the location of mutants with the $\mathrm{I}^{\mathrm{s}}$ phenotype. The mutants colored yellow surround or are within the inducer binding pocket. The surface colored red are $\mathrm{I}^{\mathrm{s}}$ mutants believed to interfere with transmission of the allosteric signal. 

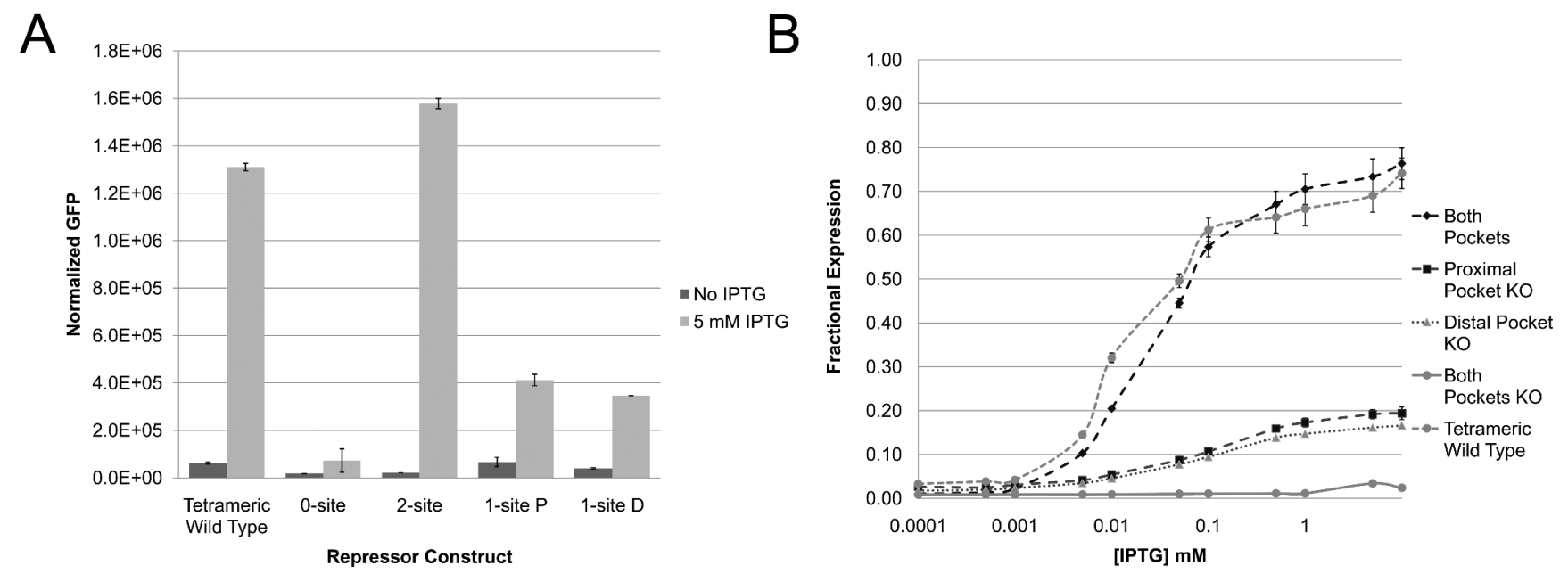

Figure 2.

Repression and Induction Data. (a)The histogram illustrates the levels GFP production in the induced and the repressed states. For comparison, GFP production is shown for the wild- type (tetrameric) repressor and the natural operator. Then next four pairs of bar graphs illustrate the level of GFP in the induced and repressed states when an R197G mutation is placed in (a) both pockets (b) neither pocket, (c) the pocket proximal to the promoter, and (d) the pocket distal to the promoter in the heterodimeric repressor. Repression by the heterodimers is with respect to the chimeric operator. (b) Response of each repressor mutant to a range of IPTG concentrations. Fractional expression defined as signal of sampled divided by signal when no repressor is present. Higher concentrations of IPTG tested produced mixed results with no increase in signal for the two site case. Each construct with and R197G mutation began inducing again, consistent with reduced IPTG affinity for that mutation. 


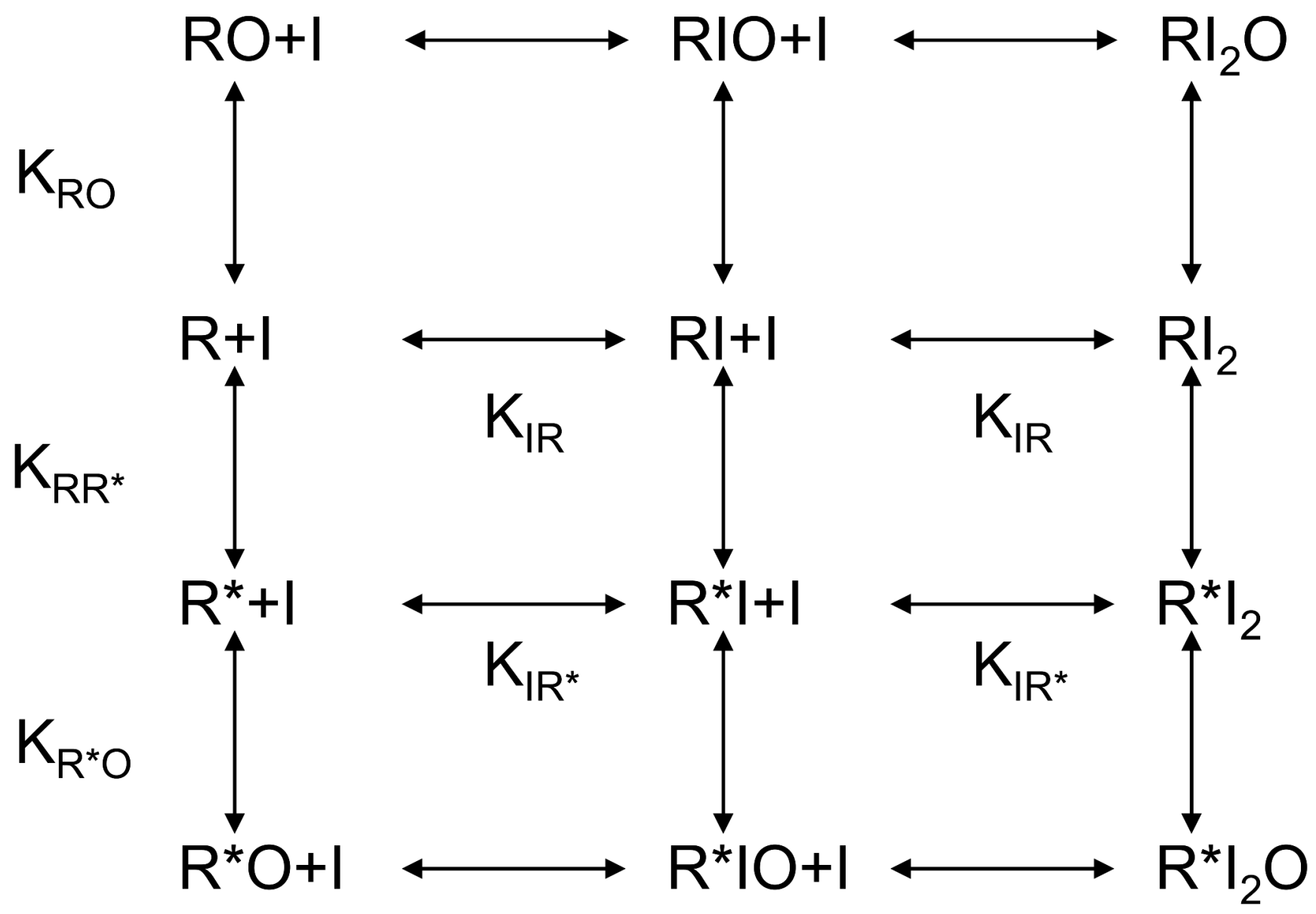

Figure 3.

A diagram illustrating the liked equibria that results from inducer binding and operator binding. $\mathrm{K}_{\mathrm{RR}} *$ is the equilibrium constant that is describes the inducer and repressed states. $\mathrm{K}_{\mathrm{IR}}$ and $\mathrm{K}_{\mathrm{IR}} *$ refer to the binding of the inducer to these two conformational states. And $\mathrm{K}_{\mathrm{RO}}$ is the equilibrium constant that describes the operator binding. 


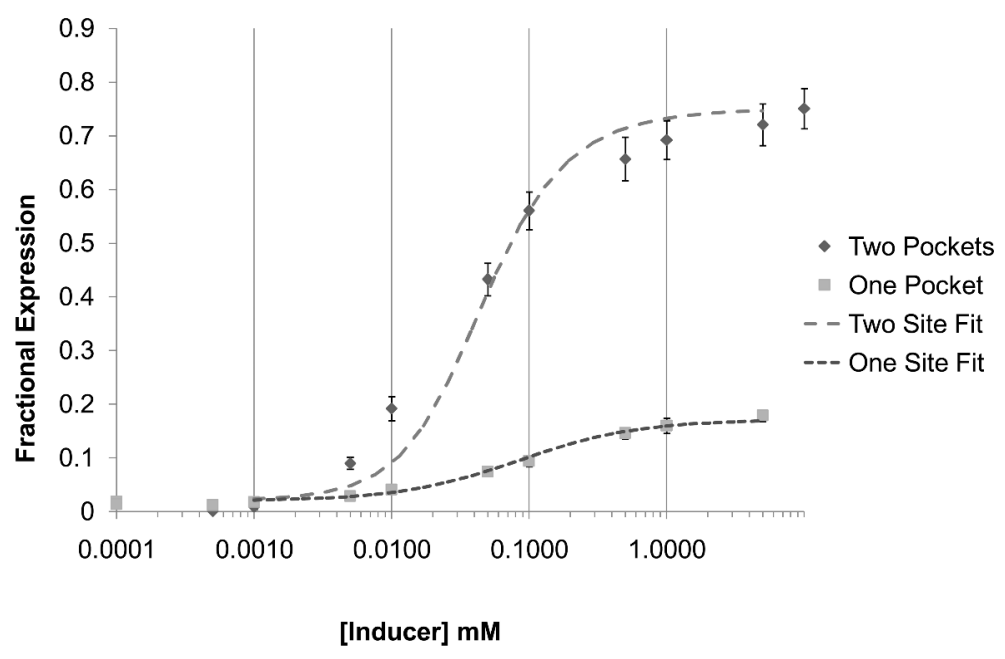

Figure 4.

The graph is a plot of the fractional induction as a function of the log of the inducer concentration for the heterodimeric repressors with a single binding pocket and two intact pockets. The curves are the expected values calculated from the model. 
A

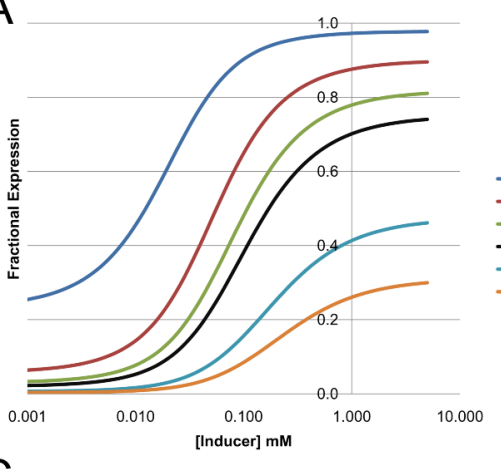

C

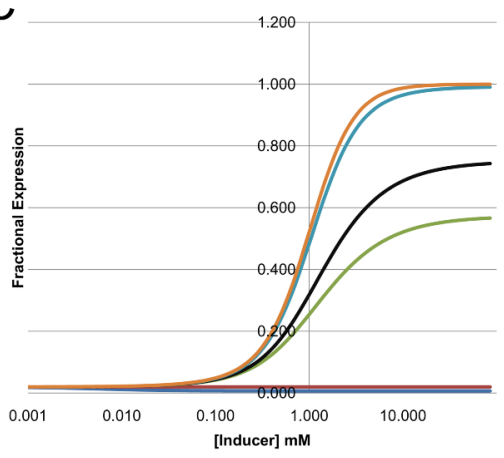

B

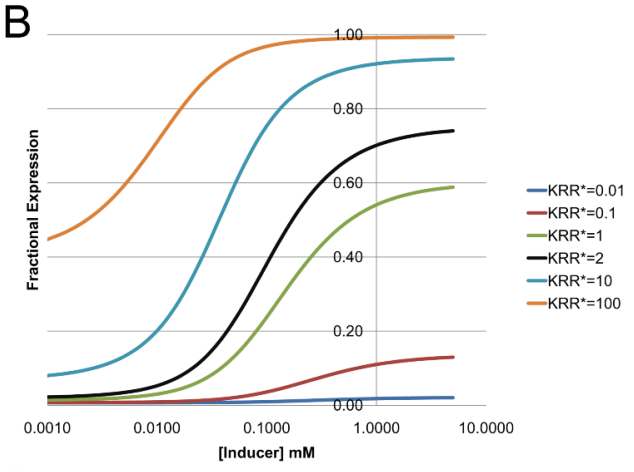

D

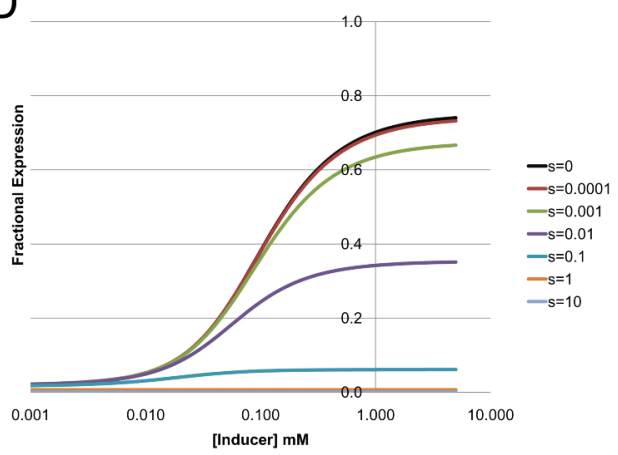

Figure 5.

Simulated plots of fractional induction with respect to inducer concentration (plotted on a log axis). Using the determined thermodynamic parameters for the repressor, theoretical plots were

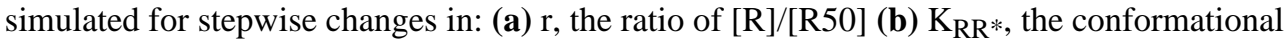
equilibrium (L), (c) $x$, the ratio of inducer binding affinities for both the active and inactive repressor conformations, and (d) s, the ratio of operator binding affinities for each of the repressor conformations. The plots shown in black correspond to the value derived from the experimental data. 


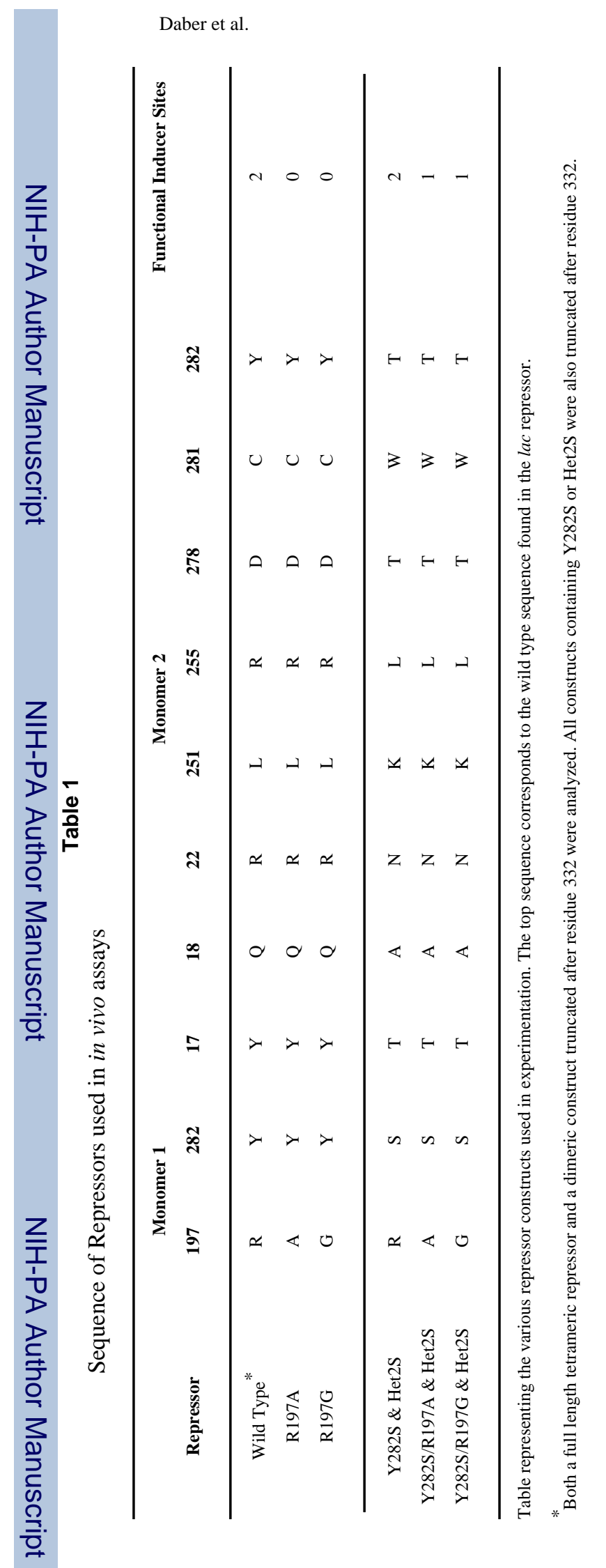

Page 21 
Daber et al.

Page 22

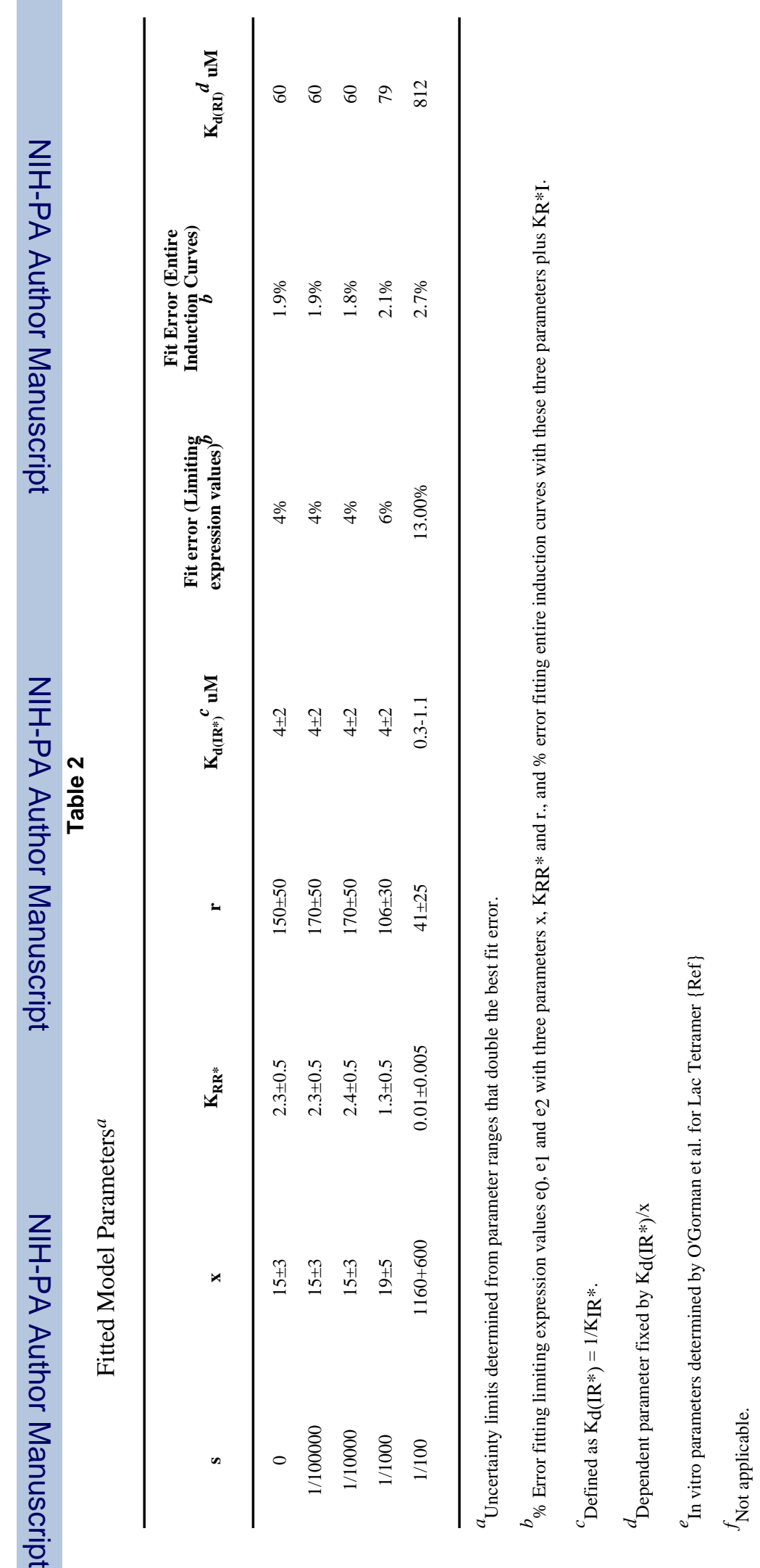

J Mol Biol. Author manuscript; available in PMC 2010 October 9. 